MS45 Measuring data quality

Chairs: Phil Evans, Alexandre Urzhumtsev

\section{MS45-01 Processing and data quality in serial femtosecond crystallography}

Thomas A. White ${ }^{1}$

1. Center for Free-Electron Laser Science, Deutsches Elektronen-Synchrotron DESY, Notkestraße 85, 22607 Hamburg, Germany

email: thomas.white@desy.de

Five years since the first publication describing serial femtosecond crystallography (SFX) [1], the technique is becoming an established technique for probing radiation-sensitive samples as well as for investigating macromolecular dynamics with high time resolution. Developments in data processing, combined with improvements to detector technology and sample delivery, mean that in 2016 we expect to be routinely able to see new information of biochemical interest, for example the electron densities of ligands absent from the structural models, in maps made using around a tenth of the data volume and measurement time which was required only a few years ago. The modern SFX analysis "toolkit" includes algorithms to resolve indexing ambiguities which arise in merohedral point groups in serial crystallography experiments [2], new methods for increasing the precision of the final intensity measurements, and much more besides. In this talk, I will detail the latest results in this area, in the context of CrystFEL, currently the most widely-used SFX data processing software [3].

[1] H. N. Chapman, P. Fromme et al. "Femtosecond X-ray protein nanocrystallography". Nature 470 (2011) p73-77. doi: 10.1038 /nature 09750

[2] W. Brehm and K. Diederichs. "Breaking the indexing ambiguity in serial crystallography". Acta Cryst. D70 (2014) p101-109.

[3] T. A. White, V. Mariani, W. Brehm, O. Yefanov, A Barty, K. R. Beyerlein, F. Chervinskii, L. Galli, C. Gati, T. Nakane, A. Tolstikova, K. Yamashita, C. H. Yoon, K. Diederichs and H. N. Chapman. "Recent developments in CrystFEL". J. Appl. Cryst. 49 (2016) p680-689. doi:10.1107/S1600576716004751

Keywords: serial femtosecond crystallography, XFEL, data processing
MS45-O2 New ways to assess the quality of single and multiple (complete and partial) $\mathrm{X}$-ray diffraction datasets

Kay Diederichs ${ }^{1}$

\section{Universität Konstanz}

email: Kay.Diederichs@uni-konstanz.de

The subject says it all. New ways of collecting data at synchrotrons and X-FEL source require new approaches to diffraction data analysis. In 2012, we described the properties of the $\mathrm{CC}_{1 / 2}$ indicator, but the opportunities to use this and related quantities have not yet been fully exploited.

Here, we describe novel ways to obtain insight into the relationships between - in particular - multiple partial datasets measured in Serial Synchrotron Crystallography experiments. It turns out that these quantities are useful also for the assessment of single datasets when these have "weak" data in certain rotation ranges of the experiment.

Keywords: accuracy, precision, partial datasets, completeness, model quality, radiation damage 